\title{
A New Expectation-Maximization Statistical Test for Case-Control Association Studies Considering Rare Variants Obtained by High-Throughput Sequencing
}

\author{
Derek Gordon $^{\mathrm{a}}$ Stephen J. Finch ${ }^{\mathrm{b}}$ Francisco De La Vega ${ }^{\mathrm{c}}$ \\ ${ }^{a}$ Department of Genetics, Rutgers University, Piscataway, N.J., ${ }^{b}$ Department of Applied Mathematics and Statistics, \\ Stony Brook University, Stony Brook, N.Y., and 'Independent researcher, San Mateo, Calif., USA
}

\section{Key Words}

Statistic $\cdot$ Genetics $\cdot$ Noncentrality parameter $\cdot$ Power $\cdot$ Misclassification - Sequence $\cdot$ Expectation-maximization • Multi-locus

\begin{abstract}
Genome-wide association studies (GWAS) have been successful in identifying common genetic variation reproducibly associated with disease. However, most associated variants confer very small risk and after meta-analysis of large cohorts a large fraction of expected heritability still remains unexplained. A possible explanation is that rare variants currently undetected by GWAS with SNP arrays could contribute a large fraction of risk when present in cases. This concept has spurred great interest in exploring the role of rare variants in disease. As the cost of sequencing continue to plummet, it is becoming feasible to directly sequence case-control samples for testing disease association including rare variants. We have developed a test statistic that allows for association testing among cases and controls using data directly from sequencing reads. In addition, our method allows for random errors in reads. We determine the probability of a true genotype call based on the observed base pair reads using the expectation-maximization algorithm. We apply the SumStat procedure to obtain a single statistic for a
\end{abstract}

group of multiple rare variant loci. We document the validity of our method through simulations. Our results suggest that our statistic maintains the correct type I error rate, even in the presence of differential misclassification for sequence reads, and that it has good power under a number of scenarios. Finally, our SumStat results show power at least as good as the maximum single locus results.

Copyright $\odot 2011$ S. Karger AG, Base

\section{Introduction}

Genome-wide association studies (GWAS) have discovered so far hundreds of common variants associated to complex disease in a reproducible manner [1]. However, only for a few of these variants causality has been established and the gene or regulatory element involved in disease etiology is known [2]. Moreover, in spite of the large sample sizes utilized so far in GWAS and their meta-analyzes, when the genetic effects of all associated variants for a given disease are accounted for, anywhere from 50 to $95 \%$ of the heritability of the trait remains yet unexplained [2]. This assessment of the state of GWAS has prompted the genetics community to consider other experimental designs that include more rare genetic variation than those accessible by SNP arrays [3-8]. There are recent studies [1,

\section{KARGER}

Fax +4161306 1234

E-Mail karger@karger.ch

www.karger.com
(C) 2011 S. Karger AG, Basel

0001-5652/11/0712-0113\$38.00/0

Accessible online at:

www.karger.com/hhe
Dr. Francisco De La Vega

27 Eastwood Drive

San Mateo, CA 94403 (USA)

Tel. +1 6509187417

E-Mail Francisco.DLV@gmail.com 
2] to suggest that variants that are rare in the population might influence disease susceptibility more strongly than the common variants studied so far. These study designs have been propelled by advances in sequencing technology that makes it possible to sequence fairly complete human genomes at a cost of thousands of dollars [9] and allows for the possibility of sequencing studies including hundreds or even thousands of individuals. In these studies a much more complete spectrum of genetic variation can in principle be tested for association, including variants that are more rare in the population (1-5\%).

Even if the cost of high-throughout sequencing continues to decrease, studies considering sequencing to completion of thousands of samples are still very expensive, and strategies to control the cost-effectiveness of these studies are necessary. Several parameters can be adjusted to fit a reasonable fixed cost for a study and yet achieve meaningful power to find association: number of samples, average depth of sequencing coverage, the error rate in the sequencing technology, and whether sequencing is carried out in individual samples or in pools of samples to save processing time and cost. Bansal et al. [8] provided an extensive review of statistical methods available for association testing with next-generation sequencing technologies. They and others conclude that the assessment of the trade-off between these variables requires considering the specific statistical test for association to be performed and the specific characteristics of sequencing data, including the error profiles of the base pair reads obtained by the high-throughput sequencer. Also, Kim et al. [10] introduced a statistical test for sequencing data considering a two-stage design that estimates the probability of including the disease-causing variant in the second stage. The authors applied this statistic to study the trade-offs of performing different types of DNA-pooling schemes. Finally, we have learned that M. Province and colleagues (Washington University, St. Louis) have recently developed a statistical method to test for association with rare variants that is robust to the 'direction' of the rare variant (i.e. the method is powerful whether the variant is more advantageous or deleterious for disease). A manuscript documenting the method is currently under review.

Here we present a new test statistic that allows for association testing among cases and controls directly using raw base pair reads instead of genotypes produced by an intermediate algorithm. Specifically, our statistic tests whether the (estimated) true genotype frequencies differ among cases and controls. Genotype frequencies are estimated using the observed sequence data. In addition, our method allows for random errors in base pair

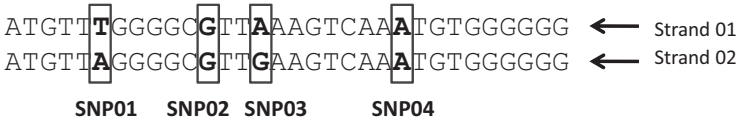

Fig. 1. Example representation of sequence reads for an individual. Top panel: A stretch of DNA sequence for an individual. The haplotype strands are labeled as '01' and '02'. As indicated, there are four SNPs in this stretch of DNA sequence. The individual is heterozygous at SNP01 (genotype T/A), homozygous at SNP02 (genotype G/G), heterozygous at SNP03 (genotype A/G) and homozygous at SNP04 (genotype A/A). Bottom panel: The sequence reads consist of random selections of one of the two strands. In this example, Strand 01 is selected 5 out of 8 times and Strand 02 is selected 3 out of 8 times. Finally, for this individual, we note that the coverage of this region of the genome is $8 \mathrm{X}$ ( 8 sequence reads).

reads that may differ among case and control populations (differential misclassification error). We determine the probability of the genotype frequencies based on the observed base pair reads using the expectation-maximization (EM) algorithm. In addition, we apply the SumStat method of Hoh, Ott and colleagues [11-15] to determine what possible power gains may be achieved over single locus methods. We document the validity of our method through simulations.

\section{Methods}

\section{Notation}

The following notation will be used in this paper.

$L_{c, i}=$ The likelihood of the true data under hypothesis $\mathrm{H}_{i}, i=$ 0 (null) or 1 (alternative); that is, this value is the likelihood of the data if every individual's genotype is determined without error.

$I()=$ Indicator function; this function takes on the value 1 when the event is true and 0 when the event is false. Throughout this work, we code the two alleles at the base pair position as 1 and 2. In addition, we specify that the 1 allele is the 'rare variant'. In figure 1 (and the text below) we provide examples of how SNP alleles may be recoded in such a manner.

$V^{(m)}=$ The total count of base pair reads for individual $m$.

$A_{n_{1}^{t}(m)}^{V(m)}=$ The true sequence-read counts for individual $m$; specifically, $n_{1}^{t}(m)$ is the true number of 1 alleles over all reads, and 
$V(m)-n_{1}^{t}(m)=n_{2}^{t}(m)$ is the true number of 2 alleles. While our method allows for the possibility that each individual has a different number of reads at a particular base pair, in this work we focus on the scenario where the number of reads is constant across all individuals. We call this number the coverage.

$A_{n_{1}(m)}^{V(m)}=$ The observed sequence-read counts for individual $m$; specifically, $n_{1}(m)$ is the observed number of 1 alleles over all reads, and $V(m)-n_{1}(m)=n_{2}(m)$ is the observed number of 2 alleles. Note that these counts may be incorrect, due to errors in individual sequence reads. Note that $n_{1}^{t}(m)+n_{2}^{t}(m)=n_{1}(m)+$ $n_{2}(m)=V(m)$. In figure 1 , we present an example of the number of observed sequence-read counts for a single individual for four SNP loci. In that figure, if we label the alleles T, G, A, and A as the 1 allele at the loci SNP01, SNP02, SNP03, and SNP04, then we have the respective counts:

$$
\begin{aligned}
& n_{1}(m)=5(\mathrm{SNP} 01), n_{1}(m)=8(\mathrm{SNP} 02) \\
& n_{1}(m)=5(\mathrm{SNP} 03), n_{1}(m)=8(\mathrm{SNP} 04) .
\end{aligned}
$$

$X_{j}^{t(m)}=$ The event that the true genotype of individual $m$ is $j$, $j=0,1$, or 2 . The value $j$ is the number of 1 alleles (rare variants) in individual $m$ 's genotype. We have used this notation in previous publications $[16,17]$.

$Y_{i}^{t(m)}=$ The event that the true affection status of individual $m$ is $i, i=0$ (case) or 1 (control). Note that in this work, we specify that the phenotype is measured without error. Therefore, individual $m$ 's true phenotype is equal to their observed phenotype. As with the true genotype notation above, we have used this notation in previous publications $[16,17]$.

$p_{i j}^{t}=\operatorname{Pr}\left(X_{j}^{t} \mid Y_{i}^{t}\right)=$ The true genotype frequency of allele $j$ with phenotype $i$ under the alternative hypothesis $\mathrm{H}_{1}$ that the genotype frequencies in cases and controls may be unequal. Written mathematically, we have:

$$
\mathrm{H}_{1}: p_{i j}^{t} \neq p_{(1-i) j}^{t} \text { for at least one } j(j=0,1 \text {, or } 2) \text { when } i=0 \text { or } 1 \text {. }
$$

$p_{j}^{t}=$ The true population frequency of the genotype $j \in\{0,1,2\}$ under the null hypothesis $\mathrm{H}_{0}$ that the genotype frequencies are equal in cases and controls. Written mathematically, we have:

$$
\mathrm{H}_{0}: p_{i j}^{t}=\operatorname{Pr}\left(X_{j}^{t} \mid Y_{i}^{t}\right)=p_{(1-i) j}^{t}=\operatorname{Pr}\left(X_{j}^{t} \mid Y_{(1-i)}^{t}\right) \text { when } i=0 \text { or } 1 \text {. }
$$

$q_{j}^{t}=\operatorname{Pr}\left(Y_{i}^{t}\right)=$ The true sampling frequency of phenotype $i$. This value is estimated directly, without error. Therefore, it remains constant through each EM algorithm update.

$Q_{1}=E\left[\ln \left(L_{c, 1}\right) \mid\right.$ Data $]=$ The expected value of the log-likelihood of the true data, conditional on the observed data under $\mathrm{H}_{1}$ (see equ. 3). This expectation is standard in the application of the EM algorithm [18].

$Q_{0}=E\left[\ln \left(L_{c, 0}\right) \mid\right.$ Data $]=$ The expected value of the log-likelihood of the true data, conditional on the observed data under $\mathrm{H}_{0}$ (see equ. 12).

$\varepsilon_{i}=$ The probability that allele $v$ is misclassified as allele $3-v$, $\nu=1,2$ for phenotype class $i$, where $i=0$ refers to a control and $i=1$ refers to a case. While we are estimating genotype frequencies, here we specify that errors occur in alleles, since the sampling unit is the sequence of base pair reads. Note that from this definition, when $\varepsilon_{i}=\varepsilon_{1-i}=\varepsilon$, the misclassification probabilities are equal in the two different directions. This is known as nondifferential misclassification [19-23]. Our statistic is more general in that it allows for differential misclassification in case-control designs. This feature is a major advantage, since differential misclas- sification has been documented to potentially cause an increase in the false-positive rate for SNP association studies [24-27].

$\tau_{m, j}=$ The posterior probability that individual $m$ 's true genotype is $j$ under $\mathrm{H}_{0}, j=0,1$, or 2 .

$\tau_{m, i, j}=$ The posterior probability that individual $m$ 's true phenotype is $i$ and their true genotype is $j$ under $\mathrm{H}_{1}, i=0,1$, and $j=$ 0,1 , or 2 .

$\ln \left(L_{0}\right)=$ The log-likelihood of the observed data under $\mathrm{H}_{0}$ that genotype frequencies are the same in the case and control groups.

$\ln \left(L_{1}\right)=$ The log-likelihood of the observed data under $\mathrm{H}_{1}$ that genotype frequencies may be different in the case and control groups.

Superscript $r,()^{(r)}=$ The $r$-th step estimate of parameters such as $\tau_{m, j}, \tau_{m, i, j}$ and $p_{i j}^{t}$ in the EM algorithm. These values are updated with each step of the EM algorithm, and are used to determine more accurate estimates of the log-likelihoods. We use the notation $\tau_{m, j}^{(r)}, \tau_{m, i, j}^{(r)}$ and $p_{i j}^{t(r)}$ to denote the $r$-th step estimates of the parameters $\tau_{m, j}, \tau_{m, i, j}$ and $p_{i j}^{t}$, respectively.

$N=$ The total number of individuals that are sequenced. Without loss of generality, we specify that the first $N_{1}$ individuals are controls, and the remaining $N_{2}=N-N_{1}$ individuals are cases.

$E M-L R T=$ The test statistic defined as $2\left(\ln \left(L_{1}\right)-\ln \left(L_{0}\right)\right)$. Here, the log-likelihoods are determined by the steps of the EM algorithm. When the difference $\left(\ln \left(L_{i}\right)\right.$ for the $(r+1)$-th step $-\ln \left(L_{i}\right)$ for the $r$-th step) is smaller than the tolerance, we say that the loglikelihood is the value at the $r$-th step. In this work, we specify a tolerance of $10^{-5}$.

\section{Likelihood Ratio Test Using the EM Algorithm}

Alternative Hypothesis $\left(\mathrm{H}_{1}\right)$

In the Results section, we shall present derivations of each loglikelihood for a given set of observed data under the alternative hypothesis $\mathrm{H}_{1}$. We shall also present closed form solutions of the posterior probabilities $\tau_{m, i, j}, i=0,1$, and $j=0,1$, or 2 .

\section{Null Hypothesis $\left(\mathrm{H}_{0}\right)$}

As with the alternative hypothesis, we write the log-likelihood of the true data under the null hypothesis $\mathrm{H}_{0}$ and shall present closed form solutions of the posterior probabilities $\tau_{m, j}, j=0,1$, or 2.

\section{Simulation Settings}

Single Locus

To evaluate the performance of the EM-LRT statistic for single loci, we perform simulations with a number of different parameter settings. These parameters and their respective settings are:

\begin{tabular}{lll} 
(a) Control true allele frequency $\alpha_{0}^{t}$ : & $0.005,0.02$ \\
(b) & Case true allele frequency $\alpha_{1}^{t}:$ & $\alpha_{0}^{t}+\delta$ \\
& (I) $\delta$ values: & $0,0.01,0.025$ \\
(c) & Coverage: & $8 \mathrm{X}, 40 \mathrm{X}$ \\
(d) & Error probabilities $\varepsilon_{\mathrm{i}}, i=0,1:$ & $0.001,0.04$ \\
(e) & Number of cases: & 1,000 \\
(f) & Number of controls: & 1,000 \\
(g) & Number of permutations per statistic: & 100 \\
(h) & Number of starting points: & 100 \\
(i) & Number of EM steps per starting point: & 100 \\
(j) & Number of replicates per vector (a-d): & 100 \\
\hline
\end{tabular}


In this work, we determine the single-locus genotype frequencies using the Hardy Weinberg Equilibrium (HWE) proportions; that is, $p_{i 0}^{t}=\left(1-\alpha_{i}^{t}\right)^{2}, p_{i 1}^{t}=2 \alpha_{i}^{t}\left(1-\alpha_{i}^{t}\right), p_{i 2}^{t}=\left(\alpha_{i}^{t}\right)^{2}, i=0,1$. For a subset of these settings (a total of 40 - see table 1 ), we simulate 100 replicates of data with 1,000 cases and 1,000 controls. As noted above, for each of the 100 replicates, we apply the EM-LRT statistic. The log-likelihoods are computed using 100 starting points for $\mathrm{H}_{0}$, with a maximum of 100 possible EM steps. Also, the final genotype frequency estimates under $\mathrm{H}_{0}$ are used as the starting values of the genotype frequencies $p_{i j}^{t}$. Specifically, $p_{i j}^{t}=p_{i}^{t}$ for the 0 -th step of the $\mathrm{H}_{1}$ estimation.

We determine $\mathrm{p}$ values asymptotically assuming a central $\chi^{2}$ with 2 degrees of freedom. In addition, we determine $\mathrm{p}$ values by permutation, performing 100 permutations of the phenotypes (item (g) above) and counting the proportion of EM-LRT statistic values determined with the permuted phenotypes that exceed the EM-LRT statistic with the observed data. Empirical type I error rate and power are determined at the 5, 1, 0.5 and $0.25 \%$ levels. All simulations are generated and analyzed using a $\mathrm{C}$ program written by one of the authors (D.G.).

\section{Multi-Locus Simulations}

To evaluate the EM-LRT statistic for multiple loci, we apply a slight variant of the SumStat procedure developed by Ott, Hoh, and colleagues $[12,13]$. While Hoh, Ott, and colleagues consider the sums of ever-increasing numbers of SNPs, in this work, we consider just the full sum. Specifically, for each of the $k$ loci, $1 \leq$ $k \leq L$, where $L$ is the number of loci in the simulation, we compute $E M-L R T(k)$, the value of the statistic at the $k$-th locus. We then compute SumStat $=\Sigma_{\mathrm{k}=1}^{L} E M-L R T(k)$. The empirical significance levels are determined through permutation. Since each locus $k$ has 50 permuted $E M-L R T(k)$ statistics associated with it, we can compute the permuted SumStat statistics for each permutation number (1-50). The empirical significance level is defined as the proportion of SumStat values that exceed the SumStat value for the observed data.

We use the following parameter settings for our multi-locus simulations:

$\begin{array}{lll}\text { (a) Number of loci: } & 3 \\ \text { (b) } & \text { First locus true allele frequency: } & \alpha_{0}^{t} \sim N\left(\mu, \sigma^{2}\right) \\ & \text { where } \mu=0.01, \sigma^{2}=10^{-6} & \\ \text { (c) } & \text { Case true allele frequency } \alpha_{1}^{t}: & \alpha_{0}^{t}+\delta \\ & \text { (I) } \delta \text { values: } & 0,0.015 \\ \text { (d) } & \text { Number of cases: } & 500 \\ \text { (e) } & \text { Number of controls: } & 500 \\ \text { (f) Number of permutations per statistic: } & 100 \\ \text { (g) Number of starting points: } & 100 \\ \text { (h) Number of EM steps per starting point: } & 100 \\ \text { (i) Number of replicates per vector }(\mathrm{a}-\mathrm{c}): & 50\end{array}$

We note that our sample sizes and replicate numbers are less for the multi-locus simulations than for the single-locus simulations. Also, we only consider 3 correlated SNPs in each simulation.

Control genotype frequencies are determined by being drawn from a normal distribution with a mean of 0.01 and a variance of $10^{-6}$. These specifications ensure that the probability that each control allele frequency will be less than 0 is less than $10^{-5}$.
Given the control genotypes for the $k$-th locus, the control genotypes for the $(k+1)$-th locus are determined using a correlation coefficient $\rho$. A full description of how the genotypes for each locus were determined is presented in the online supplementary Appendix (www.karger.com/doi/10.1159/000325590). This Appendix may also be read at: http://linkage.rockefeller.edu/derek/ EMLRT-Appendix.html, or it may be obtained by requesting a copy from the first author (D.G. E-Mail: gordon@biology.rutgers. edu).

\section{Results}

\section{Likelihood Ratio Test Using the EM Algorithm \\ Alternative Hypothesis $\left(\mathrm{H}_{1}\right)$}

Borrowing some notation from Zhou and Pan [28] (with some slight modification), we can write the loglikelihood of the true data under $\mathrm{H}_{1}$ as:

$$
\begin{aligned}
& \ln \left(L_{c, 1}\right)= \\
& \sum_{m=1}^{N} \sum_{j=0}^{2} \sum_{i=0}^{1}\left[I\left(X_{j}^{t(m)}, Y_{i}^{t(m)}\right) \times \ln \left(\operatorname{Pr}\left(X_{j}^{t(m)}, Y_{i}^{t(m)}\right)\right)\right]= \\
& \sum_{m=1}^{N} \sum_{j=1}^{2} \sum_{i=0}^{1} I\left(X_{j}^{t(m)}, Y_{i}^{t(m)}\right) \times \ln \left(p_{i j}^{t} q_{i}^{t}\right)
\end{aligned}
$$

where $I()$ is the indicator function, being 1 when $X_{j}^{t(m)}$ is the true genotype for individual $m, j=0,1$, or 2 , and $Y_{j}^{t(m)}$ is the true phenotype status of individual $m, i=0$ (control) or 1 (case). In this work, the true phenotype status is assumed to be known without error. In practice, we do not precisely know the value of $I\left(X_{j}^{t(m)}, Y_{j}^{t(m)}\right)$, since we do not know the value $X_{j}^{t(m)}$. Thus, our main interest is in the determination of the individual's genotype; the sequence data is a 'means to an end' in the sense that, with sufficient coverage, we can establish the individual's genotype with near perfect accuracy by using the EM algorithm.

Let

$$
Q_{1}=E\left[\operatorname{In}\left(L_{c, 1}\right) \mid \text { Data }\right]=\sum_{m=1}^{N} \Sigma_{j=0}^{2} \sum_{i=0}^{1} \tau_{m, i, j} \ln \left(p_{i j}^{t(m)} q_{i}^{t(m)}\right),
$$

where

$$
\begin{aligned}
\tau_{m, i, j} & =E\left[I\left(X_{j}^{t(m)}, Y_{i}^{t(m)}\right) \mid\left(A_{n_{1}(m)}^{V(m)}, Y_{i}^{t(m)}\right)\right] \\
& =\operatorname{Pr}\left(\left(X_{j}^{t(m)}, Y_{i}^{t(m)}\right) \mid\left(A_{n_{1}(m)}^{V(m)}, Y_{i}^{t(m)}\right)\right) \\
& =\frac{\operatorname{Pr}\left(A_{n_{1}(m)}^{V(m)}, X_{j}^{t(m)}, Y_{i}^{t(m)}\right)}{\operatorname{Pr}\left(A_{n_{1}(m)}^{V(m)}, Y_{i}^{t(m)}\right)} \\
& =\sum_{n_{1}^{t}(m)=0}^{V(m)} \frac{\operatorname{Pr}\left(A_{n_{1}(m)}^{V(m)}, A_{n_{1}^{t}(m)}^{V(m)}, X_{j}^{t(m)}, Y_{i}^{t(m)}\right)}{\operatorname{Pr}\left(A_{n_{1}(m)}^{V(m)}, Y_{i}^{t(m)}\right)} .
\end{aligned}
$$


Using the equations in the online supplementary Appendix, we have:

$$
\begin{aligned}
& \operatorname{Pr}\left(A_{n_{1}(m)}^{V(m)}, A_{n_{1}^{t}(m)}^{V(m)}, X_{j}^{t(m)}, Y_{i}^{t(m)}\right) \\
& =\sum_{y=\max \left(0, n_{1}^{t}(m)+n_{1}(m)-V(m)\right)}\left[\left(\begin{array}{c}
n_{1}^{t}(m) \\
y
\end{array}\right)\left(\begin{array}{c}
V(m)-n_{1}^{t}(m) \\
\left.n_{1}(m)-n_{1}^{t}(m)\right)
\end{array}\right)\right. \\
& \times\left(1-\varepsilon_{i}\right)^{V(m)-n_{1}^{t}(m)-n_{1}(m)+2 y} \varepsilon_{i} n_{1}^{t}(m)+n_{1}(m)-2 y \\
& \left.\times\left(\begin{array}{c}
V(m) \\
n_{1}^{t}(m)
\end{array}\right)\left(\frac{j}{2}\right)^{n_{1}^{t}(m)}\left(\frac{2-j}{2}\right)^{V(m)-n_{1}^{t}(m)} \times p_{i j}^{t} q_{i}^{t}\right] .
\end{aligned}
$$

Also, we have:

$$
\begin{aligned}
& \operatorname{Pr}\left(A_{n_{1}(m)}^{V(m)}, Y_{i}^{t(m)}\right) \\
& =\sum_{n_{1}^{t}(m)=0}^{V(m)} \sum_{j=0}^{2} \operatorname{Pr}\left(A_{n_{1}(m)}^{V(m)}, A_{n_{1}^{t}(m)}^{V(m)}, X_{j}^{t(m)}, Y_{i}^{t(m)}\right) \\
& =\sum_{n_{1}^{t}(m)=0}^{V(m)} \sum_{j=0}^{2}\left[\operatorname{Pr}\left(A_{n_{1}(m)}^{V(m)} \mid A_{n_{1}^{t}(m)}^{V(m)}, X_{j}^{t(m)}, Y_{i}^{t(m)}\right)\right. \\
& \left.\times \operatorname{Pr}\left(A_{n_{1}^{t}(m)}^{V(m)} \mid X_{j}^{t(m)}, Y_{i}^{t(m)}\right) \operatorname{Pr}\left(X_{j}^{t(m)} \mid Y_{i}^{t(m)}\right) \operatorname{Pr}\left(Y_{i}^{t(m)}\right)\right] \\
& =\sum_{n_{1}^{t}(m)=0}^{V(m)} \sum_{j=0}^{2}\left[\operatorname{Pr}\left(A_{n_{1}(m)}^{V(m)} \mid A_{n_{1}^{t}(m)}^{V(m)}, Y_{i}^{t(m)}\right)\right. \\
& \left.\times \operatorname{Pr}\left(A_{n_{1}^{t}(m)}^{V(m)} \mid X_{j}^{t(m)}\right) \operatorname{Pr}\left(X_{j}^{t(m)} \mid Y_{i}^{t(m)}\right) \operatorname{Pr}\left(Y_{i}^{t(m)}\right)\right] \\
& =\sum_{n_{1}^{t}(m)}^{V(m)} \sum_{j=0}^{2}\left[\operatorname{Pr}\left(A_{n_{1}(m)}^{V(m)} \mid A_{n_{1}^{t}(m)}^{V(m)}, Y_{i}^{t(m)}\right)\right. \\
& \left.\times \operatorname{Pr}\left(A_{n_{1}^{t}(m)}^{V(m)} \mid X_{j}^{t(m)}\right) \operatorname{Pr}\left(X_{j}^{t(m)} \mid Y_{i}^{t(m)}\right) \operatorname{Pr}\left(Y_{i}^{t(m)}\right)\right] \\
& =\sum_{n_{1}^{t}(m)}^{V(m)} \sum_{j=0}^{2} \sum_{y=\max \left(0, n_{1}^{t}(m)+n_{1}(m)-V(m)\right)}^{\min \left(n_{1}(m), n_{1}^{t}(m)\right)}\left[\left(\begin{array}{c}
n_{1}^{t}(m) \\
y
\end{array}\right)\left(\begin{array}{c}
V(m)-n_{1}^{t}(m) \\
n_{1}(m)-y
\end{array}\right)\right. \\
& \times(1-\varepsilon)^{V(m)-n_{1}^{t}(m)-n_{1}(m)+2 y} \varepsilon^{n_{1}^{t}(m)+n_{1}(m)-2 y} \\
& \left.\times\left(\begin{array}{c}
V(m) \\
n_{1}^{t}(m)
\end{array}\right)\left(\frac{j}{2}\right)^{n_{1}^{t}(m)}\left(\frac{2-j}{2}\right)^{V(m)-n_{1}^{t}(m)} p_{i j}^{t} q_{i}^{t}\right] .
\end{aligned}
$$

It follows that:

$$
\begin{aligned}
\tau_{m, i, j} & =\sum_{n_{1}^{t}(m)=0}^{V(m)} \frac{\operatorname{Pr}\left(A_{n_{1}(m)}^{V(m)}, A_{n_{1}^{t}(m)}^{V(m)}, X_{j}^{t(m)}, Y_{i}^{t(m)}\right)}{\operatorname{Pr}\left(A_{n_{1}(m)}^{V(m)}, Y_{i}^{t(m)}\right)} \\
& =\frac{\sum_{n_{1}^{t}(m)=0}^{V(m)} \operatorname{Pr}\left(A_{n_{1}(m),}^{V(m)}, A_{n_{1}^{t}(m)}^{V(m)}, X_{j}^{t(m)}, Y_{i}^{t(m)}\right)}{\operatorname{Pr}\left(A_{n_{1}(m)}^{V(m)}, Y_{i}^{t(m)}\right)}
\end{aligned}
$$

can be written as two expressions: equation $5 \mathrm{~A}$, when allowing for differential misclassification, and equation $5 \mathrm{~B}$, when specifying nondifferential misclassification. Due to the length of each expression, we placed equations $5 \mathrm{~A}$ and $5 \mathrm{~B}$ in online supplementary table 1 . This table may also be viewed online at: http://linkage.rockefeller.edu/derek/ EMLRT-Table01.html, or it may be obtained by requesting a copy from the first author (D.G.E-Mail: gordon@biology.rutgers.edu). From these equations we see that the determination of the $r$-th step estimates of the parameter $\tau_{m, i, j}$ amounts to the determination of the $r$-th step estimates of the parameter $p_{i j}^{t}$, since $\varepsilon_{i}$ does not appear in equation 4 . We discuss the estimates of the error rates $\varepsilon_{i}$ as the proportion of rare alleles that appear in all sequence reads, since the specification is that rare alleles have an almost 0 probability of occurring.

To compute the $r$-th step estimates of the genotype frequency parameters $p_{i j}^{t}$, we note that we may write the loglikelihood $Q_{1}$ as:

$$
\begin{aligned}
& \sum_{m=1}^{N_{1}} \sum_{j=0}^{2} \tau_{m, 0, j}^{(r-1)}\left[\ln \left(p_{0 j}^{t(r-1)}\right)+\ln \left(q_{0}^{t(r-1)}\right)\right] \\
& +\sum_{m=N_{1}+1}^{N} \sum_{j=0}^{2} \tau_{m, 1, j}^{(r-1)}\left[\ln \left(p_{1 j}^{t(r-1)}\right)+\ln \left(q_{1}^{t(r-1)}\right)\right],
\end{aligned}
$$

where the first $N_{1}$ individuals are controls, and the remaining $N_{2}=N-N_{1}$ individuals are cases. From this point forward, we drop the superscripts $(r-1)$ for clarity when deriving the $r$-th step estimates. Computing the partial derivatives $\frac{\partial Q_{1}}{\partial p_{00}^{t}}, \frac{\partial Q_{1}}{\partial p_{01}^{t}}, \frac{\partial Q_{1}}{\partial p_{02}^{t}}$, and setting each expression equal to 0 , we get:

$$
\sum_{m=1}^{N_{1}}\left(\frac{\tau_{m, 0,0}}{p_{00}^{t}}-\frac{\tau_{m, 0,2}}{p_{02}^{t}}\right)=0,
$$

and

$$
\sum_{m=1}^{N_{1}}\left(\frac{\tau_{m, 0,1}}{p_{01}^{t}}-\frac{\tau_{m, 0,2}}{p_{02}^{t}}\right)=0 .
$$

The equation corresponding to the partial derivative $\frac{\partial Q_{1}}{\partial p_{02}^{t}}$ yields the same equation as equation $6 \mathrm{~A}$.

We can rewrite equation $6 \mathrm{~A}$ as:

$$
\begin{aligned}
& 0=\sum_{m=1}^{N_{1}}\left(\frac{\tau_{m, 0,0}}{p_{00}^{t}}-\frac{\tau_{m, 0,2}}{p_{02}^{t}}\right), \\
& =\sum_{m=1}^{N_{1}}\left(\frac{\tau_{m, 0,0} \times p_{02}^{t}}{p_{00}^{t}}-\frac{\tau_{m, 0,2} \times p_{00}^{t}}{p_{02}^{t}}\right), \\
& =\sum_{m=1}^{N_{1}}\left(\frac{\tau_{m, 0,0} \times p_{02}^{t}-\tau_{m, 0,2} \times p_{00}^{t}}{p_{00}^{t} \times p_{02}^{t}}\right), \\
& =\sum_{m=1}^{N_{1}}\left(\frac{\tau_{m, 0,0}\left(1-p_{00}^{t}-p_{01}^{t}\right)-\tau_{m, 0,2} \times p_{00}^{t}}{p_{00}^{t} \times p_{02}^{t}}\right),
\end{aligned}
$$

Hum Hered 2011;71:113-125 


$$
\begin{aligned}
& =\frac{1}{p_{00}^{t} \times p_{02}^{t}} \sum_{m=1}^{N_{1}}\left(\tau_{m, 0,0}\left(1-p_{01}^{t}\right)-p_{00}^{t}\left(\tau_{m, 0,0}+\tau_{m, 0,2}\right)\right) \\
& =\frac{1}{p_{00}^{t} \times p_{02}^{t}} \sum_{m=1}^{N_{1}}\left(\tau_{m, 0,0}\left(1-p_{01}^{t}\right)-p_{00}^{t}\left(\tau_{m, 0,0}+\tau_{m, 0,2}\right)\right) \\
& =\frac{1}{p_{00}^{t} \times p_{02}^{t}} \sum_{m=1}^{N_{1}}\left(\tau_{m, 0,0}\left(1-p_{01}^{t}\right)-p_{00}^{t}\left(1-\tau_{m, 0,1}\right)\right) .
\end{aligned}
$$

The last equation is derived using the fact that $\Sigma_{j=0}^{2}$ $\tau_{m, i, j}=1$ for each $i$.

It follows that we must have:

$$
\sum_{m=1}^{N_{1}}\left(\tau_{m, 0,0}\left(1-p_{01}^{t}\right)-p_{00}^{t}\left(1-\tau_{m, 0,1}\right)\right)=0 .
$$

Using equation $6 \mathrm{~B}$, through a similar derivation we can show that:

$$
\sum_{m=1}^{N_{1}}\left(\tau_{m, 0,1}\left(1-p_{00}^{t}-p_{01}^{t}\right)-\tau_{m, 0,2} \times p_{01}^{t}\right)=0,
$$

or

$$
\sum_{m=1}^{N_{1}}\left(\tau_{m, 0,1}\left(1-p_{00}^{t}\right)-p_{01}^{t}\left(1-\tau_{m, 0,0}\right)\right)=0 .
$$

Combining equations $7 \mathrm{~A}$ and $7 \mathrm{~B}$ and using the identities $\Sigma_{j=0}^{2} \tau_{m, i, j}=1$ and $\Sigma_{j=0}^{2} p_{i j}^{t}=1$ for each $i$, we determine:

$$
p_{02}^{t(r)}=\frac{\sum_{m=1}^{N_{1}} \tau_{m, 0,2}^{(r-1)}}{N_{1}} .
$$

Using equations $6 \mathrm{~A}, 6 \mathrm{~B}$, and $8 \mathrm{~A}$, it is straightforward to show that:

$$
\begin{aligned}
& p_{01}^{t(r)}=\frac{\sum_{m=1}^{N_{1}} \tau_{m, 0,1}^{(r-1)}}{N_{1}} . \\
& p_{00}^{t(r)}=\frac{\sum_{m=1}^{N_{1}} \tau_{m, 0,0}^{(r-1)}}{N_{1}} .
\end{aligned}
$$

Using the partial derivatives $\frac{\partial Q_{1}}{\partial p_{10}^{t}}, \frac{\partial Q_{1}}{\partial p_{11}^{t}}, \frac{\partial Q_{1}}{\partial p_{12}^{t}}$, and applying the same algebra, we can show that:

$$
p_{1 j}^{t(r)}=\frac{\sum_{m=N_{1}+1}^{N} \tau_{m, 1, j}^{(r-1)}}{N_{2}},
$$

for $j=0,1$, and 2 .

Regarding $q_{i}^{t(r)}$, we have

$$
\frac{\partial Q_{1}}{\partial q_{0}^{t}}=\sum_{m=1}^{N_{1}} \sum_{j=0}^{2} \frac{\tau_{m, 0, j}}{q_{0}^{t}}-\sum_{m=N_{1}+1}^{N} \sum_{j=0}^{2} \frac{\tau_{m, 1, j}}{1-q_{0}^{t}} .
$$

Setting this equation to 0 and manipulating the terms, our solution for $q_{0}^{t(r)}$ is given by:

$$
\begin{aligned}
q_{0}^{t(r)} & =\frac{\sum_{m=1}^{N_{1}}\left(\sum_{j=0}^{2} \tau_{m, 0, j}^{(r-1)}\right)}{\sum_{m=1}^{N_{1}}\left(\sum_{j=0}^{2} \tau_{m, 0, j}\right)+\sum_{m=N_{1}+1}^{N}\left(\sum_{j=1}^{2} \tau_{m, 1, j}\right)} \\
& =\frac{\sum_{m=1}^{N_{1}}(1)}{\sum_{m=1}^{N_{1}}(1)+\sum_{m=N_{1}+1}^{N}(1)}=\frac{N_{1}}{N} .
\end{aligned}
$$

That is, the estimates of $q_{i}^{t}$ are constant and are not updated with each EM algorithm step. This result is not surprising, given that the phenotype status is known with certainty for each individual, i.e. there are no missing data.

The log-likelihood of the data under $\mathrm{H}_{1}$ is given by:

$$
\begin{aligned}
& \ln \left(L_{1}\right)=\sum_{m=1}^{N} \sum_{i=0}^{1} I\left(Y_{i}^{t(m)}\right) \ln \left[\operatorname{Pr}\left(A_{n_{1}(m)}^{V(m)}, Y_{i}^{t(m)}\right)\right] \\
& =\sum_{m=1}^{N_{1}} \ln \left[\operatorname{Pr}\left(A_{n_{1}(m)}^{V(m)}, Y_{0}^{t(m)}\right)\right] \\
& +\sum_{m=N_{1}+1}^{N} \ln \left[\operatorname{Pr}\left(A_{n_{1}(m)}^{V(m)}, Y_{1}^{t(m)}\right)\right] \\
& =\sum_{m=1}^{N_{1}} \ln
\end{aligned}
$$

$\left[\sum_{n_{1}^{t}(m)=0}^{V(m)} \sum_{j=0}^{2} \sum_{y=\max \left(0, n_{1}^{t}(m)+n_{1}(m)-V(m)\right)}^{\min \left(n_{1}(m), n_{1}^{t}(m)\right)}\left[\left(\begin{array}{c}n_{1}^{t}(m) \\ y\end{array}\right)\left(\begin{array}{c}V(m)-n_{1}^{t}(m) \\ n_{1}(m)-y\end{array}\right)\right.\right.$ $\times\left(1-\varepsilon_{0}\right)^{V(m)-n_{1}^{t}(m)-n_{1}(m)+2 y} \varepsilon_{0} n_{1}^{t}(m)+n_{1}(m)-2 y$

$\left.\left.\times\left(\begin{array}{c}V(m) \\ n_{1}^{t}(m)\end{array}\right)\left(\frac{j}{2}\right)^{n_{1}^{t}(m)}\left(\frac{2-j}{2}\right)^{V(m)-n_{1}^{t}(m)} p_{i j}^{t} q_{i}^{t}\right]\right]$

$+\sum_{m=N_{1}+1}^{N} \ln$

$\left[\sum_{n_{1}^{t}(m)}^{V(m)} \sum_{j=0}^{2} \sum_{y=\max \left(0, n_{1}^{t}(m)+n_{1}(m)-V(m)\right)}^{\min \left(n_{1}(m), n_{1}^{t}(m)\right)}\left[\left(\begin{array}{c}n_{1}^{t}(m) \\ y\end{array}\right)\left(\begin{array}{c}V(m)-n_{1}^{t}(m) \\ n_{1}(m)-y\end{array}\right)\right.\right.$ $\times\left(1-\varepsilon_{1}\right)^{V(m)-n_{1}^{t}(m)-n_{1}(m)+2 y} \varepsilon_{1} n_{1}^{t}(m)+n_{1}(m)-2 y$ $\left.\left.\times\left(\begin{array}{c}V(m) \\ n_{1}^{t}(m)\end{array}\right)\left(\frac{j}{2}\right)^{n_{1}^{t}(m)}\left(\frac{2-j}{2}\right)^{V(m)-n_{1}^{t}(m)} p_{i j}^{t} q_{i}^{t}\right]\right]$,

where $I\left(Y_{i}^{t(m)}\right)$ is the indicator function for the phenotype of the $m$-th individual; more specifically, $I\left(Y_{i}^{t(m)}\right)=1$ if the phenotype of the $m$-th individual is $i$, and 0 otherwise. 
Also, the $r$-th step of the parameters $I\left(p_{i j}^{t(r)}\right)$ satisfy the condition that:

$\mid\left(\ln \left(L_{1}\right)\right.$ using estimates $\left.p_{i j}^{t(r)}\right)-$

$\left(\ln \left(L_{1}\right)\right.$ using estimates $\left.p_{i j}^{t(r-1)}\right) \mid<10^{-5}$.

\section{Null Hypothesis $\left(H_{0}\right)$}

We write the log-likelihood of the true data under $\mathrm{H}_{0}$ as:

$$
\begin{aligned}
\ln \left(L_{c, 0}\right) & =\sum_{m=1}^{N} \sum_{j=0}^{2} \sum_{i=0}^{1}\left[I\left(X_{j}^{t(m)}, Y_{i}^{t(m)}\right)\right. \\
& \left.\times \ln \left(\operatorname{Pr}\left(X_{j}^{t(m)}, Y_{i}^{t(m)}\right)\right)\right] \\
& =\sum_{m=1}^{N} \sum_{j=1}^{2} \sum_{i=0}^{1} I\left(X_{j}^{t(m)}, Y_{i}^{t(m)}\right) \times \ln \left(p_{j}^{t} q_{i}^{t}\right),
\end{aligned}
$$

where (as in equ. 3) $I()$ is the indicator function, being 1 when $X_{j}^{t(m)}$ is the true genotype for individual $m, j=0,1$, or 2 , and $Y_{i}^{t(m)}$ is the true phenotype status of individual $m, i=0$ (control) or 1 (case). The difference between this $\log$-likelihood and the true log-likelihood for $\mathrm{H}_{1}$ is that in equation 11 the genotype frequency $p_{j}^{t}$ does not depend upon the phenotype.

Similar to above, let:

$$
\begin{aligned}
Q_{0} & =E\left[\ln \left(L_{c, 0}\right) \mid \text { Data }\right] \\
& =\sum_{m=1}^{N} \sum_{j=0}^{2} \sum_{i=0}^{1} \tau_{m, j} \ln \left(p_{j}^{t(m)} q_{i}^{t(m)}\right),
\end{aligned}
$$

where

$$
\begin{aligned}
\tau_{m, j} & =E\left[I\left(X_{j}^{t(m)}\right) \mid\left(A_{n_{1}(m)}^{V(m)}\right)\right], \\
= & \operatorname{Pr}\left(X_{j}^{t(m)} \mid A_{n_{1}(m)}^{V(m)}\right), \\
= & \frac{\operatorname{Pr}\left(A_{n_{1}(m)}^{V(m)}, X_{j}^{t(m)}\right)}{\operatorname{Pr}\left(A_{n_{1}(m)}^{V(m)}\right)} \\
= & \sum_{n_{1}^{t}(m)=0}^{V(m)} \frac{\operatorname{Pr}\left(A_{n_{1}(m)}^{V(m)}, A_{n_{1}^{t}(m)}^{V(m)}, X_{j}^{t(m)}\right)}{\operatorname{Pr}\left(A_{n_{1}(m)}^{V(m)}\right)} .
\end{aligned}
$$

Using the equations in the online supplementary Appendix, we have:

$$
\begin{aligned}
& \operatorname{Pr}\left(A_{n_{1}(m)}^{V(m)}, A_{n_{1}^{t}(m)}^{V(m)}, X_{j}^{t(m)}\right) \\
& =\sum_{i=0}^{1} \operatorname{Pr}\left(A_{n_{1}(m)}^{V(m)}, A_{n_{1}^{t}(m)}^{V(m)}, X_{j}^{t(m)}, Y_{i}^{t(m)}\right) \\
& =\sum_{i=0}^{\min \left(n_{1}(m), n_{1}^{t}(m)\right)} \sum_{y=\max \left(0, n_{1}^{t}(m)+n_{1}(m)-V(m)\right)}\left[\left(\begin{array}{c}
n_{1}^{t}(m) \\
y
\end{array}\right)\left(\begin{array}{c}
V(m)-n_{1}^{t}(m) \\
n_{1}(m)-y
\end{array}\right)\right.
\end{aligned}
$$

$$
\begin{aligned}
& \times\left(1-\varepsilon_{i}\right)^{V(m)-n_{1}^{t}(m)-n_{1}(m)+2 y} \varepsilon_{i} n_{1}^{t}(m)+n_{1}(m)-2 y \\
& \left.\times\left(\begin{array}{c}
V(m) \\
n_{1}^{t}(m)
\end{array}\right)\left(\frac{j}{2}\right)^{n_{1}^{t}(m)}\left(\frac{2-j}{2}\right)^{V(m)-n_{1}^{t}(m)} p_{j}^{t}\right] .
\end{aligned}
$$

This expression simplifies to:

$$
\begin{aligned}
& \sum_{y=\max \left(0, n_{1}^{t}(m)+n_{1}(m)-V(m)\right)}^{\min \left(n_{1}(m), n_{1}^{t}(m)\right)}\left[\left(\begin{array}{c}
n_{1}^{t}(m) \\
y
\end{array}\right)\left(\begin{array}{c}
V(m)-n_{1}^{t}(m) \\
n_{1}(m)-y
\end{array}\right)\right. \\
& \times\left(1-\varepsilon_{i}\right)^{V(m)-n_{1}^{t}(m)-n_{1}(m)+2 y_{\varepsilon_{i}} n_{1}^{t}(m)+n_{1}(m)-2 y} \\
& \left.\times\left(\begin{array}{c}
V(m) \\
n_{1}^{t}(m)
\end{array}\right)\left(\frac{j}{2}\right)^{n_{1}^{t}(m)}\left(\frac{2-j}{2}\right)^{V(m)-n_{1}^{t}(m)} p_{j}^{t}\right] .
\end{aligned}
$$

Under nondifferential misclassification, we have $\varepsilon_{0}=\varepsilon_{1}=$ $\varepsilon$, so equation $13 \mathrm{~A}$ simplifies to:

$$
\begin{aligned}
& \operatorname{Pr}\left(A_{n_{1}(m)}^{V(m)}, A_{n_{1}^{t}(m)}^{V(m)}, X_{j}^{t(m)}\right)=\sum_{i=0}^{1}\left(q_{i}^{t}\right) \\
& \times \sum_{y=\max \left(0, n_{1}^{t}(m)+n_{1}(m)-V(m)\right)}\left[\left(\begin{array}{c}
n_{1}^{t}(m) \\
y
\end{array}\right)\left(\begin{array}{c}
V(m)-n_{1}^{t}(m) \\
n_{1}(m)-y
\end{array}\right)\right. \\
& \times(1-\varepsilon)^{V(m)-n_{1}^{t}(m)-n_{1}(m)+2 y} \varepsilon^{n_{1}^{t}(m)+n_{1}(m)-2 y} \\
& \left.\times\left(\begin{array}{c}
V(m) \\
n_{1}^{t}(m)
\end{array}\right)\left(\frac{j}{2}\right)^{n_{1}^{t}(m)}\left(\frac{2-j}{2}\right)^{V(m)^{-} n_{1}^{t}(m)} p_{j}^{t}\right], \\
& =\quad \sum^{\min \left(n_{1}(m), n_{1}^{t}(m)\right)} \\
& \quad\left[\left(\begin{array}{c}
n_{1}^{t}(m) \\
y=\max \left(0, n_{1}^{t}(m)+n_{1}(m)-V(m)\right)
\end{array}\right)\left(\begin{array}{c}
V(m)-n_{1}^{t}(m) \\
n_{1}(m)-y
\end{array}\right)\right. \\
& \times(1-\varepsilon)^{V(m)-n_{1}^{t}(m)-n_{1}(m)+2 y} \varepsilon^{n_{1}^{t}(m)+n_{1}(m)-2 y} \\
& \left.\times\left(\begin{array}{c}
V(m) \\
n_{1}^{t}(m)
\end{array}\right)\left(\frac{j}{2}\right)^{n_{1}^{t}(m)}\left(\frac{2-j}{2}\right)^{V(m)-n_{1}^{t}(m)} p_{j}^{t}\right] .
\end{aligned}
$$

Also, we have:

$$
\begin{aligned}
& \operatorname{Pr}\left(A_{n_{1}(m)}^{V(m)}\right)=\sum_{i=0}^{1} \operatorname{Pr}\left(A_{n_{1}(m)}^{V(m)}, Y_{i}^{t(m)}\right) \\
= & \sum_{n_{1}^{t}(m)=0}^{V(m)} \sum_{j=0}^{2} \sum_{i=0}^{1} \operatorname{Pr}\left(A_{n_{1}(m)}^{V(m)}, A_{n_{1}^{t}(m)}^{V(m)}, X_{j}^{t(m)}, Y_{i}^{t(m)}\right) \\
= & \sum_{n_{1}^{t}(m)=0}^{V(m)} \sum_{j=0}^{2} \sum_{i=0}^{1} \sum_{y=\max \left(0, n_{1}^{t}(m)+n_{1}(m)-V(m)\right)}^{\min \left(n_{1}(m), n_{1}^{t}(m)\right)}\left[\begin{array}{c}
n_{1}^{t}(m) \\
y
\end{array}\right)\left(\begin{array}{c}
V(m)-n_{1}^{t}(m) \\
n_{1}(m)-y
\end{array}\right) \\
\times & \left(1-\varepsilon_{i}\right)^{V(m)-n_{1}^{t}(m)-n_{1}(m)+2 y} \varepsilon_{i} n_{1}^{t}(m)+n_{1}(m)-2 y \\
\times & \left.\left(\begin{array}{c}
V(m) \\
n_{1}^{t}(m)
\end{array}\right)\left(\frac{j}{2}\right)^{n_{1}^{t}(m)}\left(\frac{2-j}{2}\right)^{V(m)-n_{1}^{t}(m)} p_{j}^{t} q_{i}^{t}\right],
\end{aligned}
$$


or under nondifferential misclassification:

$$
\begin{aligned}
& \operatorname{Pr}\left(A_{n_{1}(m)}^{V(m)}\right)=\sum_{n_{1}^{t}(m)=0}^{V(m)} \sum_{j=0}^{2} \sum_{i=0}^{1} \operatorname{Pr}\left(A_{n_{1}(m)}^{V(m)}, A_{n_{1}^{t}(m)}^{V(m)}, X_{j}^{t(m)}, Y_{i}^{t(m)}\right) \\
& =\sum_{n_{1}^{t}(m)=0}^{V(m)} \sum_{j=0}^{2} \sum_{i=0}^{1} \min \left(n_{1}(m), n_{1}^{t}(m)\right) \\
& \times(1-\varepsilon)^{V(m)-n_{1}^{t}(m)-n_{1}(m)+2 y} \sum_{\varepsilon^{n_{1}^{t}(m)+n_{1}(m)-2 y}}\left[\left(\begin{array}{c}
n_{1}^{t}(m) \\
y
\end{array}\right)\left(\begin{array}{c}
V(m)-n_{1}^{t}(m) \\
n_{1}(m)-y
\end{array}\right)\right. \\
& \left.\times\left(\begin{array}{c}
V(m) \\
n_{1}^{t}(m)
\end{array}\right)\left(\frac{j}{2}\right)^{n_{1}^{t}(m)}\left(\frac{2-j}{2}\right)^{V(m)-n_{1}^{t}(m)} p_{j}^{t} q_{i}^{t}\right] .
\end{aligned}
$$

Using these equations, we can determine closed form solutions of the probabilities $\tau_{m, j}$. They are: equation $15 \mathrm{~A}$, when allowing for differential misclassification, and equation $15 \mathrm{~B}$, when specifying nondifferential misclassification. Due to the length of the expressions, we placed equations $15 \mathrm{~A}$ and $15 \mathrm{~B}$ in online supplementary table 1.

From equations $15 \mathrm{~A}$ and $15 \mathrm{~B}$ we see that the determination of the $r$-th step estimates of $\tau_{m, j}$ amounts to the determination of the $r$-th step estimates of the parameter $p_{j}^{t}$, since $\varepsilon_{i}$ does not appear in equation 12 . We already noted this above, with equation 5 .

To compute the $r$-th step estimates of the genotype frequency parameters $p_{j}^{t}$, we note that we may write the loglikelihood $Q_{0}$ as:

$$
\begin{aligned}
& \sum_{m=1}^{N} \sum_{j=0}^{2} \sum_{i=0}^{1}\left[\tau_{m, j}^{(r-1)} \ln \left(p_{j}^{t(r-1)}\right)\right]+ \\
& \sum_{m=1}^{N_{1}} \sum_{j=0}^{2} \tau_{m, j}^{(r-1)}\left[\ln \left(q_{0}^{t(r-1)}\right)\right]+ \\
& \sum_{m=N_{1}+1}^{N} \sum_{j=0}^{2} \tau_{m, j}^{(r-1)}\left[\ln \left(q_{1}^{t(r-1)}\right)\right] .
\end{aligned}
$$

Here, as above, the first $N_{1}$ individuals are controls, and the remaining $N_{2}=N-N_{1}$ individuals are cases. From this point forward, we drop the superscripts $(r-1)$ for clarity when deriving the $r$-th step estimates. Computing the partial derivatives $\frac{\partial Q_{0}}{\partial p_{0}^{t}}, \frac{\partial Q_{0}}{\partial p_{1}^{t}}, \frac{\partial Q_{0}}{\partial p_{2}^{t}}$, and setting each expression equal to 0 , we get:

$$
2 \sum_{m=1}^{N}\left(\frac{\tau_{m, 0}}{p_{0}^{t}}-\frac{\tau_{m, 2}}{1-p_{0}^{t}-p_{1}^{t}}\right)=0,
$$

and

$$
2 \sum_{m=1}^{N}\left(\frac{\tau_{m, 1}}{p_{1}^{t}}-\frac{\tau_{m, 2}}{1-p_{0}^{t}-p_{1}^{t}}\right)=0 .
$$

Using the same algebra we did with equations $6 \mathrm{~A}$, $6 \mathrm{~B}, 7 \mathrm{~A}$ and $7 \mathrm{~B}$ and using the identities $\Sigma_{j=0}^{2} \tau_{m, j}=1$ and $\sum_{j=0}^{2} p_{j}^{t}=1$ we can show:

$$
p_{j}^{t(r)}=\frac{\sum_{m=1}^{N} \tau_{m, j}^{(r-1)}}{N},
$$

for all $j$.

Regarding $q_{i}^{t(r)}$, we have (as with $Q_{1}$ above),

$$
\frac{\partial Q_{0}}{\partial q_{0}^{t}}=\sum_{m=1}^{N_{1}} \sum_{j=0}^{2} \frac{\tau_{m, j}}{q_{0}^{t}}-\sum_{m=N_{1}+1}^{N} \sum_{j=0}^{2} \frac{\tau_{m, j}}{1-q_{0}^{t}}
$$

Setting this equation to 0 and manipulating the terms, our solution for $q_{0}^{t(r)}$ is given by:

$$
\begin{aligned}
q_{0}^{t(r)} & =\frac{\sum_{m=1}^{N_{1}}\left(\sum_{j=0}^{2} \tau_{m, j}\right)}{\sum_{m=1}^{N_{1}}\left(\sum_{j=0}^{2} \tau_{m, j}\right)+\sum_{m=N_{1}+1}^{N}\left(\sum_{j=1}^{2} \tau_{m, j}\right)} \\
& =\frac{\sum_{m=1}^{N_{1}}(1)}{\sum_{m=1}^{N_{1}}(1)+\sum_{m=N_{1}+1}^{N}(1)}=\frac{N_{1}}{N} .
\end{aligned}
$$

As above, the estimates of $q_{i}^{t}$ are constant and are not updated with each EM algorithm step.

The log-likelihood of the data under $\mathrm{H}_{0}$ is given by:

$$
\begin{aligned}
& \ln \left(L_{0}\right)=\sum_{m=1}^{N} \sum_{n_{1}^{t}(m)=0}^{V(m)} \sum_{j=0}^{2} \sum_{i=0}^{1} \ln \left[\operatorname{Pr}\left(A_{n_{1}(m)}^{V(m)}, Y_{i}^{t(m)}\right)\right] \\
& =\sum_{m=1}^{N_{1}} \sum_{n_{1}^{t}(m)}^{V(m)} \sum_{j=0}^{2} \\
& {\left[\operatorname { l n } \left[\operatorname{Pr}\left(A_{n_{1}(m)}^{V(m)} \mid A_{n_{1}^{t}(m)}^{V(m)}, X_{j}^{t(m)}, Y_{0}^{t(m)}\right) \operatorname{Pr}\left(A_{n_{1}^{t}(m)}^{V(m)} \mid X_{j}^{t(m)}, Y_{0}^{t(m)}\right)\right.\right.} \\
& \left.\left.\times \operatorname{Pr}\left(X_{j}^{t(m)} \mid Y_{0}^{t(m)}\right) \operatorname{Pr}\left(Y_{0}^{t(m)}\right)\right]\right] \\
& +\sum_{m=N_{1}+1}^{N} \sum_{n_{1}^{t}(m)=0}^{V(m)} \sum_{j=0}^{2} \\
& {\left[\operatorname { l n } \left[\operatorname{Pr}\left(A_{n_{1}(m)}^{V(m)} \mid A_{n_{1}^{t}(m)}^{V(m)}, X_{j}^{t(m)}, Y_{1}^{t(m)}\right) \operatorname{Pr}\left(A_{n_{1}^{t}(m)}^{V(m)} \mid X_{j}^{t(m)}, Y_{1}^{t(m)}\right)\right.\right.} \\
& \left.\left.\times \operatorname{Pr}\left(X_{j}^{t(m)} \mid Y_{1}^{t(m)}\right) \operatorname{Pr}\left(Y_{1}^{t(m)}\right)\right]\right] \text {, } \\
& =\sum_{m=1}^{N_{1}} \sum_{n_{1}^{t}(m)}^{V(m)} \sum_{j=0}^{2}[\ln \\
& \sum_{y=\max \left(0, n_{1}^{t}(m)+n_{1}(m)-V(m)\right)}^{\min \left(n_{1}(m), n_{1}^{t}(m)\right)}\left[\left(\begin{array}{c}
n_{1}^{t}(m) \\
y
\end{array}\right)\left(\begin{array}{c}
V(m)-n_{1}^{t}(m) \\
n_{1}(m)-y
\end{array}\right)\right. \\
& \times\left(1-\varepsilon_{0}\right)^{\left.V(m)-n_{1}^{t}(m)-n_{1}(m)+2 y_{\varepsilon_{0}} n_{1}^{t}(m)+n_{1}(m)-2 y\right]}
\end{aligned}
$$




$$
\begin{aligned}
& \left.\left.\times\left(\begin{array}{c}
V(m) \\
n_{1}^{t}(m)
\end{array}\right)\left(\frac{j}{2}\right)^{n_{1}^{t}(m)}\left(\frac{2-j}{2}\right)^{V(m)-n_{1}^{t}(m)} p_{j}^{t(r)} q_{0}^{t}\right]\right] \\
& +\sum_{m=N_{1}+1}^{N} \sum_{n_{1}^{t}(m)=0}^{V(m)} \sum_{j=0}^{2}[\ln \\
& \sum_{y=\max \left(0, n_{1}^{t}(m)+n_{1}(m)-V(m)\right)}^{\min \left(n_{1}(m), n_{1}^{t}(m)\right)}\left[\left(\begin{array}{c}
n_{1}^{t}(m) \\
y
\end{array}\right)\left(\begin{array}{c}
V(m)-n_{1}^{t}(m) \\
n_{1}(m)-y
\end{array}\right)\right. \\
& \left.\times\left(1-\varepsilon_{1}\right)^{V(m)-n_{1}^{t}(m)-n_{1}(m)+2 y} \varepsilon_{1} n_{1}^{t}(m)+n_{1}(m)-2 y\right] \\
& \left.\times\left(\begin{array}{c}
V(m) \\
n_{1}^{t}(m)
\end{array}\right)\left(\frac{j}{2}\right)^{n_{1}^{t}(m)}\left(\frac{2-j}{2}\right)^{V(m)-n_{1}^{t}(m)} p_{j}^{t(r)} q_{1}^{t}\right],
\end{aligned}
$$

where the $r$-th step of the parameters $p_{j}^{t(r)}$ satisfy the condition that:

$$
\begin{aligned}
& \mid\left(\ln \left(L_{0}\right) \text { using estimates } p_{j}^{t(r)}\right)- \\
& \quad\left(\ln \left(L_{0}\right) \text { using estimates } p_{j}^{t(r-1)}\right) \mid<10^{-5} .
\end{aligned}
$$

\section{Test Statistic EM-LRT}

As noted above in the Notation section, the test statistic EM-LRT is defined as:

$$
E M-L R T=2\left[\ln \left(L_{1}\right)-\ln \left(L_{0}\right)\right]
$$

The log-likelihoods are determined by the steps of the EM algorithm. When the difference

$\mid\left(\ln \left(L_{k}\right)\right.$ using estimates $\left.p_{i j}^{t(r)}\right)-$

$\left(\ln \left(L_{k}\right)\right.$ using estimates $\left.p_{i j}^{t(r)}\right) \mid<10^{-5}$,

we specify that the $\log$-likelihood $\ln \left(L_{k}\right)$ at the $r$-th step is the value used in the test statistic (equ. 19). (Note that this inequality is for $\mathrm{H}_{1}$, since the parameters of interest for $\mathrm{H}_{0}$ are $p_{j}^{t(r)}$.) In this work, we use a tolerance of $10^{-5}$. Note, however, that different tolerance values may be used. Computationally, the smaller the tolerance, the more EM steps are necessary to determine the log-likeli$\operatorname{hood} \ln \left(L_{i}\right)$.

\section{Single-Locus Simulations}

Results of the single-locus simulations are presented in table 1 . The asymptotic results are computed specifying a central $\chi^{2}$ distribution with 2 degrees of freedom (from 3 genotypes), and the permutation results are computed by performing 100 permutations of the case and control status for each simulated data set.
Considering the values in this table, we can draw a number of conclusions. First, the EM-LRT statistic appears to maintain the correct type I error rate (simulations in which $\delta=0$ ). Using the BINOM program with the value $0 / 100=0.0$, we compute that the $95 \%$ confidence interval for the value 0 is $(0.0,0.0295)$. Thus, the only simulation that suggests that $E M-L R T$ is slightly conservative is the one in which coverage is $8 \mathrm{X}$, the control MAF is 0.005 , and the case and control error rates are 0.001 . For all other null simulations, our results suggest that EM-LRT maintains the correct type I error rate. Of particular note is that fact that EM-LRT maintains the correct rate even when the case and control error rates are different (differential misclassification rates). Recently, several authors [24-27, 29-31] have documented that there can be substantial inflation in the type I error rate in the presence of differential misclassification. It is a useful feature of the $E M-L R T$ statistic that this phenomenon does not occur.

An additional result is that power determined by permutation is usually larger than power determined by asymptotic theory. This result is not surprising, given that we specified HWE for the control genotype frequencies, with very small minor allele frequencies. That is, the 'Cochran Condition' [32] of an expected cell count of 5 is not achieved in these data sets, suggesting that the use of 2 degrees of freedom is too conservative.

A final and important result stems from a comparison of the $40 \mathrm{X}$ versus $8 \mathrm{X}$ coverage results when all other parameters are the same. In the situations where $\delta>0$ (power), the $40 \mathrm{X}$ coverage results are usually larger than the $8 \mathrm{X}$ coverage results for the same significance levels (both asymptotic and permutation results). This observation is most pronounced for the simulation settings: $\alpha_{0}^{t}=0.005$, $\delta=0.01, \varepsilon_{1}=0.001, \varepsilon_{0}=0.04$. For these settings, the $40 \mathrm{X}$ power at the $5 \%$ level (permutation) is 0.92 , while the $8 \mathrm{X}$ power at the $5 \%$ level (permutation) is 0.30 , a difference of 0.62 . What accounts for this difference in power? In results not shown, the parameter estimates for the two different coverages are nearly the same. The major difference between the simulations is that convergence is much more rapid for the $40 \mathrm{X}$ coverage than for the $8 \mathrm{X}$ coverage. That is, the $\mathrm{H}_{0}$ equality:

$$
\begin{aligned}
& \mid\left(\ln \left(L_{0}\right) \text { using estimates } p_{j}^{t(r)}\right)- \\
& \quad\left(\ln \left(L_{0}\right) \text { using estimates } p_{j}^{t(r-1)}\right) \mid<10^{-5} .
\end{aligned}
$$

is satisfied for a median value of $r=3$ steps with $40 \mathrm{X}$ coverage as compared to a median value of $r=16$ steps with $8 \mathrm{X}$ coverage. Similarly, the $\mathrm{H}_{1}$ inequality:

\footnotetext{
$\mid\left(\ln \left(L_{1}\right)\right.$ using estimates $\left.p_{i j}^{t(r)}\right)-$

$\left(\ln \left(L_{1}\right)\right.$ using estimates $\left.p_{i j}^{t(r)}\right) \mid<10^{-5}$
} 
Table 1. Single-locus simulation results of the EM-LRT statistic

\begin{tabular}{|c|c|c|c|c|c|c|c|c|c|c|c|c|}
\hline Coverage & $\delta$ & $\alpha_{0}^{t}$ & $\varepsilon_{1}$ & $\varepsilon_{0}$ & $5 \%$ & $1 \%$ & $0.5 \%$ & $0.25 \%$ & $5 \%$ & $1 \%$ & $0.5 \%$ & $0.25 \%$ \\
\hline \multirow{3}{*}{8} & \multirow{2}{*}{0.000} & 0.005 & 0.040 & 0.040 & 0.01 & 0.00 & 0.00 & 0.00 & 0.03 & 0.00 & 0.00 & 0.00 \\
\hline & & 0.020 & 0.001 & 0.001 & 0.02 & 0.00 & 0.00 & 0.00 & 0.04 & 0.00 & 0.00 & 0.00 \\
\hline & 0.010 & 0.005 & 0.001 & 0.001 & 0.77 & 0.55 & 0.44 & 0.33 & 0.89 & 0.65 & 0.58 & 0.51 \\
\hline \multirow[t]{16}{*}{40} & \multirow[t]{5}{*}{0.000} & \multirow[t]{4}{*}{0.005} & \multirow[t]{2}{*}{0.001} & 0.001 & 0.01 & 0.00 & 0.00 & 0.00 & 0.01 & 0.01 & 0.01 & 0.01 \\
\hline & & & & 0.040 & 0.02 & 0.00 & 0.00 & 0.00 & 0.03 & 0.01 & 0.01 & 0.00 \\
\hline & & & \multirow[t]{2}{*}{0.040} & 0.001 & 0.01 & 0.00 & 0.00 & 0.00 & 0.01 & 0.01 & 0.01 & 0.01 \\
\hline & & & & 0.040 & 0.02 & 0.00 & 0.00 & 0.00 & 0.06 & 0.01 & 0.00 & 0.00 \\
\hline & & $\overline{0.020}$ & 0.001 & 0.001 & 0.01 & 0.00 & 0.00 & 0.00 & 0.02 & 0.00 & 0.00 & 0.00 \\
\hline & \multirow[t]{8}{*}{0.010} & \multirow[t]{4}{*}{0.005} & \multirow[t]{2}{*}{0.001} & 0.001 & 0.79 & 0.55 & 0.50 & 0.43 & 0.88 & 0.75 & 0.72 & 0.56 \\
\hline & & & & 0.040 & 0.81 & 0.58 & 0.47 & 0.37 & 0.92 & 0.69 & 0.65 & 0.54 \\
\hline & & & \multirow[t]{2}{*}{$\overline{0.040}$} & 0.001 & 0.80 & 0.59 & 0.47 & 0.37 & 0.93 & 0.71 & 0.63 & 0.48 \\
\hline & & & & 0.04 & 0.80 & 0.54 & 0.50 & 0.41 & 0.89 & 0.65 & 0.61 & 0.50 \\
\hline & & \multirow[t]{4}{*}{0.020} & \multirow[t]{2}{*}{0.001} & 0.001 & 0.47 & 0.23 & 0.16 & 0.14 & 0.54 & 0.34 & 0.28 & 0.21 \\
\hline & & & & 0.040 & 0.36 & 0.19 & 0.15 & 0.11 & 0.49 & 0.24 & 0.22 & 0.16 \\
\hline & & & 0.040 & 0.001 & 0.47 & 0.22 & 0.16 & 0.14 & 0.52 & 0.32 & 0.27 & 0.21 \\
\hline & & & & 0.040 & 0.42 & 0.27 & 0.18 & 0.14 & 0.51 & 0.32 & 0.28 & 0.18 \\
\hline & 0.025 & 0.005 & 0.001 & 0.001 & 1.00 & 1.00 & 1.00 & 1.00 & 1.00 & 1.00 & 1.00 & 1.00 \\
\hline & & & & 0.040 & 1.00 & 1.00 & 1.00 & 1.00 & 1.00 & 1.00 & 1.00 & 1.00 \\
\hline & & & 0.040 & 0.001 & 1.00 & 1.00 & 1.00 & 1.00 & 1.00 & 1.00 & 1.00 & 0.99 \\
\hline
\end{tabular}

$\alpha_{0}^{t}=$ True control minor allele frequency. $\delta=$ Value used to determine true case minor allele frequency. Specifically, true case minor allele frequency $=\alpha_{1}^{t}=\alpha_{0}^{t}+\delta$. $\varepsilon_{i}=$ True error rate for controls $(i=0)$ and cases $(i=1)$.

is satisfied for a median value of $r=2$ steps with $40 \mathrm{X}$ coverage as compared to a median value of $r=7$ steps with $8 \mathrm{X}$ coverage. These results suggest that faster convergence leads to more accuracy and more power for the EM$L R T$ with $40 \mathrm{X}$ coverage, since there is less 'variance' in the estimate of the final log-likelihoods for the 40X coverage situation than for the $8 \mathrm{X}$ coverage simulations. In fact, the variances in the final $\log$-likelihoods for $\mathrm{H}_{0}$ and $\mathrm{H}_{1}$ for the $1008 \mathrm{X}$ simulations are 63,515 and 63,280 , respectively, as compared with 19,972 and 19,509 for the 40X simulations. That is, the variances for the $8 \mathrm{X}$ coverage simulations are more than 3 -fold greater.

\section{Multi-Locus Simulations}

We present results of the multi-locus simulations under $\mathrm{H}_{1}(\delta=0.015)$ in figure 2 . In this figure, empirical power for single loci is computed as follows: for each simulation, we have case-control data simulated for 3 loci. We compute the EM-LRT statistic permutation p value for each locus, order the p values from smallest to largest, with $i=1$ corresponding to the smallest $\mathrm{p}$ value and $i=3$ corresponding to the largest, determine the maximum $i$ for which $p_{i} \leq i / 3 \alpha$, where $\alpha$ is the specified significance level $(0.05,0.01,0.005$, and 0.0025 in these simulations; see below for a further description of the inequality), de- 


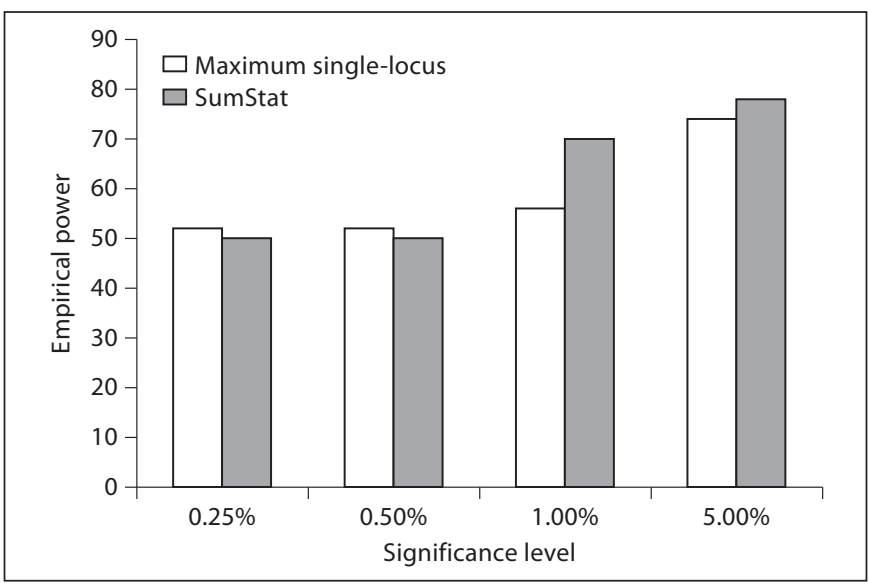

Fig. 2. Results of multi-locus simulations. The empirical power of the maximum single-locus method and the SumStat method for 3 loci that each have a pair-wise correlation coefficient of 0.90 . Empirical power for the maximum single-locus method is described in the Results section (Multi-Locus Simulations) and empirical power for the SumStat procedure at a given significance level $(5,1,0.5,0.25 \%)$ is defined as the proportion of SumStat statistics (out of 50 simulations) whose empirical $p$ value is less than the given significance level.

fine $k=\max (i)$, and declare the loci in each simulation from 1 to $k$ as significant (note, it is possible for a given simulation that no $i$ satisfies the inequality). Next, for each of the 3 loci, we compute the proportion of simulations (out of 50) for which each locus has been declared significantly associated with the phenotype; we define this value as the power of the respective locus. Finally, for each significance level, we determine the locus with the largest power and report this value in figure 2. The inequality above is used to determine the False Discovery Rate [33], which has been shown to be more powerful than the Bonferroni correction when controlling for multiple testing. Also, Benjamini and Yekutieli [34] documented that this inequality is appropriate when multiple tests are positively correlated, as is the situation in these simulations.

For the SumStat procedure, as mentioned in the Methods section, the empirical significance level (either type I error or power) is defined as the proportion of values that exceed the value for the observed data when data are simulated under a particular hypothesis (either null or alternative, respectively). Studying figure 2, we see that at the higher significance levels $(1 \%, 5 \%)$, empirical power for the SumStat procedure is larger than that of the maximum single-locus method. This difference is greatest at the $1 \%$ level, where the Sum Stat's empirical power is $70 \%$, and the maximum single-locus's power is $56 \%$. With the relatively small number of simulations, we do have an overlap of the $95 \%$ confidence intervals for all significance levels, suggesting there is no clear advantage of one method over the other based on these simulations. Still, we can say that it appears that no power is lost using the SumStat procedure. Also, we conjecture that power gains will be greater when there are more loci involved in defining a trait, since the SumStat method elegantly addresses the multiple testing issue by performing a joint test of all loci at a time.

We note that we do not have a figure for the empirical type I error rate simulations $(\delta=0)$. The reason for this is that all simulations yielded values of 0 at all significance levels. That is, every permutation $p$ value was greater than 0.05 in our simulations. However, using the BINOM program, we note that the $95 \%$ confidence interval for 50 simulations is $0.00-0.058$, suggesting that every simulation $\mathrm{p}$ value contains all thresholds in the $95 \%$ confidence intervals. We plan to perform more simulations with larger permutation $\mathrm{p}$ values. This is work in progress.

\section{Discussion}

There are several advantages to our test statistic. They are: (1) the fact that the test statistic can test for association with observed errors in the data; (2) as with other EM algorithm statistics (e.g. [28]), we can estimate the probability that an individual with a particular phenotype has a particular genotype. This information is incorporated in the terms $\tau_{m, j}$ and $\tau_{m, i, j}$. (3) As with other recently published statistics [10], we can compute the $E M-L R T$ statis-tic using individuals with differing sequence coverages. Since we can analyze raw read data directly rather than genotype calls provided by intermediate algorithms that are very sensitive to individual read coverage, this allows for flexible designs where a tradeoff is made between individual read coverage and sample size. (4) As noted in the Results, convergence of the EM algorithm is rather rapid; and (5) our test statistic appears to be robust to differential misclassification error in sequence reads.

With a few notable exceptions, case-control genetic association studies have not produced a large of number of causal susceptibility variants. One hypothesis to explain this observation is that there are multiple loci, each of large effect but with small minor allele frequency [2] that work 
in concert to produce a disease phenotype. Sequencing technologies are well suited to detect the loci with small minor allele frequencies. To address the issue of multiple loci, we applied the SumStat approach proposed by Ott and colleagues [for sums of test statistics, see 11-13, 15, 35]; this approach may well be ideal for detecting association of multiple loci within a functional unit such as a gene with a disease phenotype. Recent work by Zhou and Pan [28] documented that sums of heterogeneity statistics for casecontrol data were more powerful than individual test statistics when multiple loci were involved in determining a phenotype. We plan to apply our SumStat work with the $E M-L R T$ in a similar fashion to determine the power gain, if any, of sums of the EM-LRT statistic over multiple loci as compared with individual locus $E M-L R T$ values for numerous different simulation parameter settings.

Much of the work here is inspired by the recent work of Kim et al. [10], in particular the flexibility in allowing each individual to have variable read coverage. While our work is motivated by Kim et al.'s work, it is not directly comparable. In their paper [10], the authors consider a two-stage design, in which data in the first stage is pooled sequence data. In contrast, our work is of a one-stage design, where sequence data is directly analyzed.
A final advantage of our test statistic is that we can use classical mathematical statistics theory to determine the noncentrality parameter of the statistic. In this way, we can determine power and sample size calculations for any genetic model parameter settings and any significance level. An example where we applied this theory was in our LRTAE test statistic [see ref. 17,36]. This is an advantage because hundreds if not thousands of power or sample size calculations can be computed in a short time, so researchers can know a range of such values over parameters such as basepair allele frequency settings. This concept has been applied in software such as PAWE-3D [37] and CaTS [38].

\section{Acknowledgements}

The authors gratefully acknowledge Dr. Jürg Ott, who graciously invited them to submit this work to this special issue of Human Heredity honoring him on the occasion of his 70th birthday. In addition, the authors most gratefully acknowledge the work of two anonymous reviewers, whose comments helped to significantly improve a previous version of the paper. Last, and certainly not least, we are extremely grateful to Dr. Wonkuk Kim, who determined errors in our calculations of conditional sequence probabilities and provided us with the correct formulas.

\section{References}

1 Manolio TA: Finding the missing heritability of complex diseases. Nature 2009;461:747-753.

12 Maher B: Personal genomes: the case of the missing heritability. Nature 2008;456:18-21.

3 Li B, Leal SM: Methods for detecting associations with rare variants for common diseases: application to analysis of sequence data. Am J Hum Genet 2008;83:311-321.

4 Li B, Leal SM: Discovery of rare variants via sequencing: implications for the design of complex trait association studies. PLoS Genet 2009;5:e1000481.

5 Zhang L, Pei YF, Li JA, Papasian CJ, Deng HW: Efficient utilization of rare variants for detection of disease-related genomic regions. PLoS One 2010;5:e14288.

6 Li Y, Byrnes AE, Li MY: To identify associations with rare variants, just WHaIT: weighted haplotype and imputation-based tests. Am J Hum Genet 2010;87:728-735.

7 Hoffmann TJ, Marini NJ, Witte JS: Comprehensive approach to analyzing rare genetic variants. PLoS One 2010;5:e13584.

8 Bansal V, Libiger O, Torkamani A, Schork NJ: Statistical analysis strategies for association studies involving rare variants. Nat Rev Genet 2010;11:773-785.
9 Netterwald J: The USD 1,000 genome: coming soon? Drug Discovery \& Development, Advantage Business Media Science Group 2010;13:14-15.

10 Kim SY, Li Y, Guo Y, Li R, Holmkvist J, Hansen T, Pedersen O, Wang J, Nielsen R: Design of association studies with pooled or unpooled next-generation sequencing data. Genet Epidemiol 2010;34:479-491.

11 Hoh J, Ott J: A train of thoughts on gene mapping. Theor Popul Biol 2001;60:149-153.

12 Hoh J, Ott J: Mathematical multi-locus approaches to localizing complex human trait genes. Nat Rev Genet 2003;4:701-709.

13 Hoh J, Wille A, Ott J: Trimming, weighting, and grouping SNPs in human case-control association studies. Genome Res 2001;11: 2115-2119.

14 Ott J, Hoh J: Statistical approaches to gene mapping. Am J Hum Genet 2000;67:289-294.

15 Wille A, Hoh J, Ott J: Sum statistics for the joint detection of multiple disease loci in case-control association studies with SNP markers. Genet Epidemiol 2003;25:350359.

16 Gordon D, Haynes C, Yang Y, Kramer PL, Finch SJ: Linear trend tests for case-control genetic association that incorporate random phenotype and genotype misclassification error. Genet Epidemiol 2007;31:853-870.
17 Gordon D, Yang Y, Haynes C, Finch SJ, Mendell NR, Brown AM, Haroutunian V: Increasing power for tests of genetic association in the presence of phenotype and/or genotype error by use of double-sampling. Stat Appl Genet Mol Biol 2004;3:Article 26.

18 Dempster AP, Laird NM, Rubin DB: Maximum likelihood from incomplete data via the EM algorithm. J Roy Statist Soc B 1977; 39:1-38.

19 Bross I: Misclassification in $2 \times 2$ tables. Biometrics 1954;10:478-486.

20 Mote VL, Anderson RL: An investigation of the effect of misclassification on the properties of $\chi^{2}$-tests in the analysis of categorical data. Biometrika 1965;52:95-109.

21 Cochran WG: Errors of measurement in statistics. Technometrics 1968;10:637-666.

$\checkmark 22$ Gordon D, Finch SJ, Nothnagel M, Ott J: Power and sample size calculations for casecontrol genetic association tests when errors are present: application to single nucleotide polymorphisms. Hum Hered 2002;54:22-33.

23 Gordon D, Finch SJ: Consequences of error; in Dunn MJ, Jorde LB, Little PFR, Subramaniam S (eds): Encyclopedia of Genetics, Genomics, Proteomics and Bioinformatics. Hoboken, J. Wiley and Sons, 2006. 
24 Moskvina V, Craddock N, Holmans P, Owen MJ, O’Donovan MC: Effects of differential genotyping error rate on the type I error probability of case-control studies. Hum Hered 2006;61:55-64.

25 Clayton DG, Walker NM, Smyth DJ, Pask R, Cooper JD, Maier LM, Smink LJ, Lam AC, Ovington NR, Stevens HE, Nutland S, Howson JM, Faham M, Moorhead M, Jones HB, Falkowski M, Hardenbol P, Willis TD, Todd JA: Population structure, differential bias and genomic control in a large-scale, case-control association study. Nat Genet 2005;37:1243-1246.

26 Plagnol V, Cooper JD, Todd JA, Clayton DG A method to address differential bias in genotyping in large-scale association studies. PLoS Genet 2007;3:e74.

27 Londono D, Haynes C, De La Vega FM, Finch SJ, Gordon D: A cost-effective statistical method to correct for differential genotype misclassification when performing casecontrol genetic association. Hum Hered 2010;70:102-108.
28 Zhou H, Pan W: Binomial mixture modelbased association tests under genetic heterogeneity. Ann Hum Genet 2009;73:614-630.

29 Ahn K, Gordon D, Finch SJ: Increase of rejection rate in case-control studies with the differential genotyping error rates. Stat Appl Genet Mol Biol 2009;8:Article 25.

30 Cheng KF, Lin WJ: The effects of misclassification in studies of gene-environment interactions. Hum Hered 2009;67:77-87.

- 31 Marquard V, Beckmann L, Heid IM, Lamina C, Chang-Claude J: Impact of genotyping errors on the type I error rate and the power of haplotype-based association methods. BMC Genet 2009; 10:3.

32 Cochran WG: Some methods for strengthening the common $\chi^{2}$ tests. Biometrics 1954; 10:417-451.

33 Benjamini Y, Hochberg Y: Controlling the false discovery rate: a practical and powerful approach to multiple testing. J R Statist Soc B 1995;57:289-300.
34 Benjamini Y, Yekutieli D: The control of the false discovery rate in multiple testing under dependency. Ann Stat 2001;29:1165-1188.

35 Ott J, Hoh J: Set association analysis of SNP case-control and microarray data. J Comput Biol 2003; 10:569-574.

36 Ji F, Yang Y, Haynes C, Finch SJ, Gordon D: Computing asymptotic power and sample size for case-control genetic association studies in the presence of phenotype and/or genotype misclassification errors. Stat Appl Genet Mol Biol 2005;4:Article 37.

37 Gordon D, Haynes C, Blumenfeld J, Finch SJ: PAWE-3D: visualizing power for association with error in case-control genetic studies of complex traits. Bioinformatics 2005;21: 3935-3937.

38 Skol AD, Scott LJ, Abecasis GR, Boehnke M: Joint analysis is more efficient than replication-based analysis for two-stage genomewide association studies. Nat Genet 2006;38: 209-213.

\section{Erratum}

In the article by Gordon et al., 'A New Expectation-Maximization Statistical Test for Case-Control Association Studies Considering Rare Variants Obtained by HighThroughput Sequencing' [Hum Hered 2011;71:113-125], an author name has been misspelled: Francisco De La Vega has to be Francisco M. De La Vega. 